\title{
Zonas susceptibles de invasión por parte del pez león en el Pacífico mexicano
}

\section{Areas susceptible to lionfish invasion in the Mexican Pacific}

\author{
Héctor Reyes-Bonilla ${ }^{1} \&$ David Petatán-Ramírez ${ }^{1 *}$
}

\begin{abstract}
RESUMEN
El pez león Pterois volitans/miles, ha invadido el Atlántico occidental, pero está ausente del Pacífico oriental a pesar de que las condiciones oceanográficas parecen ser adecuadas para su establecimiento. Este trabajo propone áreas probables de invasión de Pterois spp. en la costa oeste de México, a partir de modelos de nicho ecológico y de distribución potencial, basados en datos de ocurrencia de la especie (1985-2018) y capas ambientales (2002-2017). Para la base de datos ambiental, se utilizaron rasters de temperatura, salinidad, clorofila $a$ y velocidad de corrientes marinas en valores promedio, máximos y mínimos en el nivel superficial, además, el promedio de la temperatura y salinidad en el nivel de fondo marino y el tipo de costa predominante. Los resultados indicaron que la salinidad del fondo, el tipo de costa y la temperatura promedio en superficie aportaron $65 \%$ de la contribución en el modelo de distribución. Para conocer el impacto de la posible llegada de Pterois spp. sobre la biodiversidad, se realizó una intersección espacial entre el área pronosticada con potencial para la invasión con la distribución geográfica de peces costeros y las zonas de pesca. De acuerdo con el análisis espacial, la llegada de Pterois spp. podría afectar a casi 200 especies de peces de las cuales, varias son endémicas e incluyen algunas de importancia comercial. El impacto podría ser mayor, si se considera que el pez león es depredador de invertebrados como jaibas y camarones, las cuales son pesquerías importantes en la región.
\end{abstract}

Palabras clave: Distribución potencial de especies, especies invasoras, golfo de California, modelo de nicho, Pterois volitans/miles

\footnotetext{
ABSTRACT

Lionfish Pterois volitans/miles has invaded the western Atlantic, but is absent in the eastern tropical Pacific, even though the oceanographic conditions seem to be adequate for its establishment. This paper proposes probable Pterois spp invasion areas on the western coast of Mexico, based on ecological niche and potential distribution models, as well as species occurrence data (1985-2018) and environmental layers (2002-2017). For the environmental database, rasters of temperature, salinity, chlorophyll $a$ and velocity of sea currents were used at average, maximum,

1 Universidad Autónoma de Baja California Sur. Departamento Académico de Biología Marina. Carretera al Sur 5.5., C.P 23080, La Paz, BCS, México.

hreyes@uabcs.mx, dpetatan@uabcs.mx*

ORCID: https://orcid.org/0000-0003-2593-9631, https://orcid.org/0000-0002-0173-8950
} 
and minimum values at the surface level, as well as average temperature and salinity at the seabed level and the type of predominant coast. Results indicated that bottom salinity, type of coast, and average surface temperature contributed $65 \%$ to the distribution model. To learn the impact of the possible invasion of Pterois spp. on local biodiversity, a spatial intersection was made between the predicted area with potential for invasion with the geographic distribution of coastal fish and the fishing areas. According to the spatial analysis, the arrival of Pterois spp. could affect almost 200 species, several of which are endemic and of commercial importance. The impact could be even greater, considering that the species is a predator of invertebrates such as crayfish and shrimp, which are important fisheries in the region.

Keywords: Species distribution model, Invasive species, Gulf of California, Niche model, Pterois volitans/miles

\section{INTRODUCCIÓN}

El pez león abarca el complejo de especies Pterois volitans/miles. Es nativo del Pacífico occidental y el océano Índico, y ha ganado relevancia internacional gracias a su notable éxito como especie invasora, que le ha permitido colonizar casi todos los mares tropicales del planeta (Bariche et al. 2013; Crocetta et al. 2015). Este organismo ha podido aclimatarse a zonas arrecifales con condiciones ambientales muy diversas debido a sus características biológicas que incluyen un amplio espectro alimenticio, alta tasa reproductiva, y la presencia de espinas con veneno que le ayudan a disminuir la mortalidad por depredación (Bernal et al. 2014). Pterois spp. fue introducido de manera artificial a la costa oriental de los Estados Unidos en 1980 y para la primera década del siglo XXI había llegado a las Bermudas, al margen continental del mar Caribe, y hasta el noreste del golfo de México (Whitfield et al. 2002; Schofield, 2009). Posteriormente se estableció al sur del golfo de México (Santander-Monsalvo et al. 2012), invadió también al mar Mediterráneo (Bariche et al. 2013), y las costas de Brasil (Ferreira et al. 2015).

En la actualidad solo hay dos regiones tropicales del mundo donde Pterois volitans/miles no ha sido capaz de irrumpir: la costa occidental de África y el Pacífico oriental tropical (Schofield, 2010). Esta última abarca desde el golfo de California (GC; México) hasta el norte de Perú, y en ella el pez no se ha establecido a pesar de la presencia de arrecifes coralinos y rocosos (Glynn et al. 2017) que son hábitats propicios para la colonización, por lo que, dada la afinidad de hábitat, Williams et al. (2015) manifestaron preocupación sobre los efectos de la llegada de Pterois spp. a la costa oeste de los Estados Unidos. Johnston y Purkis (2014) argumentaron que no puede descartarse la eventual introducción del pez hacia la costa oeste del continente americano, ya sea causada por liberación de ejemplares 
por acción humana, el transporte de individuos en masas de detritos flotantes desde el Pacífico central, o el paso de larvas o adultos en agua de lastre desde el Caribe y a través del canal de Panamá. Finalmente, MacIsaac et al. (2016) combinaron información sobre la calidad del ambiente y la posibilidad de arribo de larvas en agua de lastre y apoyan la posibilidad de éxito de inmigración de larvas de Pterois spp. al Pacífico americano.

Los trabajos previamente citados concluyeron que la probabilidad más alta de riesgo de establecimiento del pez león se presenta en el GC y aguas adyacentes en México, y en las islas Galápagos, Ecuador, y ello abre una interrogante sobre las zonas específicas de esos países que pudieran ser más susceptibles a la invasión (si esta llegase a ocurrir). Dado que la literatura coincide en indicar que las costas del Pacífico de México estarían en alto riesgo, es importante advertir con anticipación cuáles serían las secciones costeras de esta región donde sería más factible la entrada de Pterois spp, y posteriormente hacer inferencias sobre los posibles impactos ecológicos y económicos de su llegada al Pacífico mexicano.

Una forma de estimar los sitios potenciales de establecimiento de una especie invasora es el uso de modelos de nicho ecológico y distribución potencial de especies (Peterson et al. 2011; Leidenberger et al. 2015). Estas metodologías han sido aplicadas para conocer las zonas posibles de inserción de algunos taxa como las algas Caulerpa taxifolia y Codium fragile en el Caribe y Atlántico norte (Johnston \& Purkis, 2012; Tyberghein et al. 2012) y el pez Fistularia commersonii en el Mediterráneo (Azzurro et al. 2013). La técnica se ha usado también en el caso de Pterois spp. (Johnston \& Purkis, 2011; 2014; Bariche et al. 2013) con el fin de detectar futuras zonas de ocurrencia en la escala mundial.

Johnston \& Purkis (2014) analizaron las posibles zonas de ocurrencia de Pterois spp. en el Pacífico oriental tropical con base en modelos de dispersión de partículas, combinados con parámetros poblacionales de la especie e información sobre su tolerancia térmica y batimétrica. Los autores simularon la liberación de larvas de Pterois spp. para determinar las zonas donde podrían llegar a establecerse y concluyeron que el mayor peligro lo presenta la región de Nayarit-Jalisco en el Pacífico central de México, y la costa de Panamá. Este enfoque puede ser adecuado pues la deriva larval representa la base de los eventos de conectividad marina, y juega un papel predominante en la colonización de especies hacia nuevas regiones (García-Molinos et al. 2016), aunque también depende en gran medida, de la calidad del hábitat que permita el asentamiento de larvas y posteriormente la reproducción exitosa de los adultos (Gaston, 2003). 
Johnston y Purkis (2014) también indicaron que el potencial de éxito de invasión de Pterois spp. al Pacífico americano no debe ser alto debido a las condiciones ambientales de esa región (alta productividad primaria y baja cobertura coralina), y porque la conectividad larval se vería dificultada por el complejo patrón de corrientes, que limitaría su transporte. Sin embargo, la alta productividad causada por presencia de surgencias o aportes fluviales no ha excluido las poblaciones naturales de Pterois en zonas costeras del océano Índico, y la inexistencia de arrecifes coralinos en la costa oriental de los Estados Unidos o el mar Mediterráneo tampoco han detenido la colonización (Kletou et al. 2016). Por otra parte, la conectividad dentro del GC y zonas cercanas como bahía de Banderas es un hecho, así como la que se presenta entre América Central y el sur de México (Lessios \& Baums, 2017), esto gracias al patrón predominante de corrientes de sur a norte (Fig. 1A; Fiedler $\&$ Talley, 2006). Además, la presencia de giros en la región central y norte de $\mathrm{GC}$, dependiendo la dirección de los vientos, favorece el reclutamiento entre la zona este y oeste de dicho mar interior (Montaño-Cortés \& Marinone, 2016).

Con base en los argumentos ofrecidos, puede decirse que el enfoque utilizado por Johnston y Purkis (2014) solo abarca una parte del problema, y aunque los resultados de MacIsaac et al. (2016) también aportan un antecedente importante que define un escenario potencial basado en la tolerancia de la especie a la temperatura y a la posibilidad de transportación por agua de lastre y tráfico marítimo, se requiere más información y el empleo de diversos tipos de herramientas para tener una mejor idea del potencial de invasión de Pterois volitans/miles al Pacífico americano. Para aportar en este sentido, el objetivo del presente trabajo fue detectar sitios potenciales de establecimiento de Pterois spp. en el Pacífico mexicano, a partir de un análisis de su nicho ecológico con procedimientos de máxima entropía. En el trabajo se generaron modelos de distribución potencial para pronosticar donde están los sitios con características ambientales idóneas para el organismo de interés. Finalmente se discuten cuáles son las especies, zonas arrecifales y pesqueras que podrían verse afectadas si Pterois spp. llegara a establecerse.

\section{MATERIALES Y MÉTODOS}

Para realizar la presente investigación se creó un modelo de distribución potencial con el software MaxEnt, ver. 3.3.3 (Phillips et al. 2006), el cual construye representaciones del nicho ecológico que utiliza registros georreferenciados de la ocurrencia de las especies combinados con datos ambientales. A partir de estos insumos, 


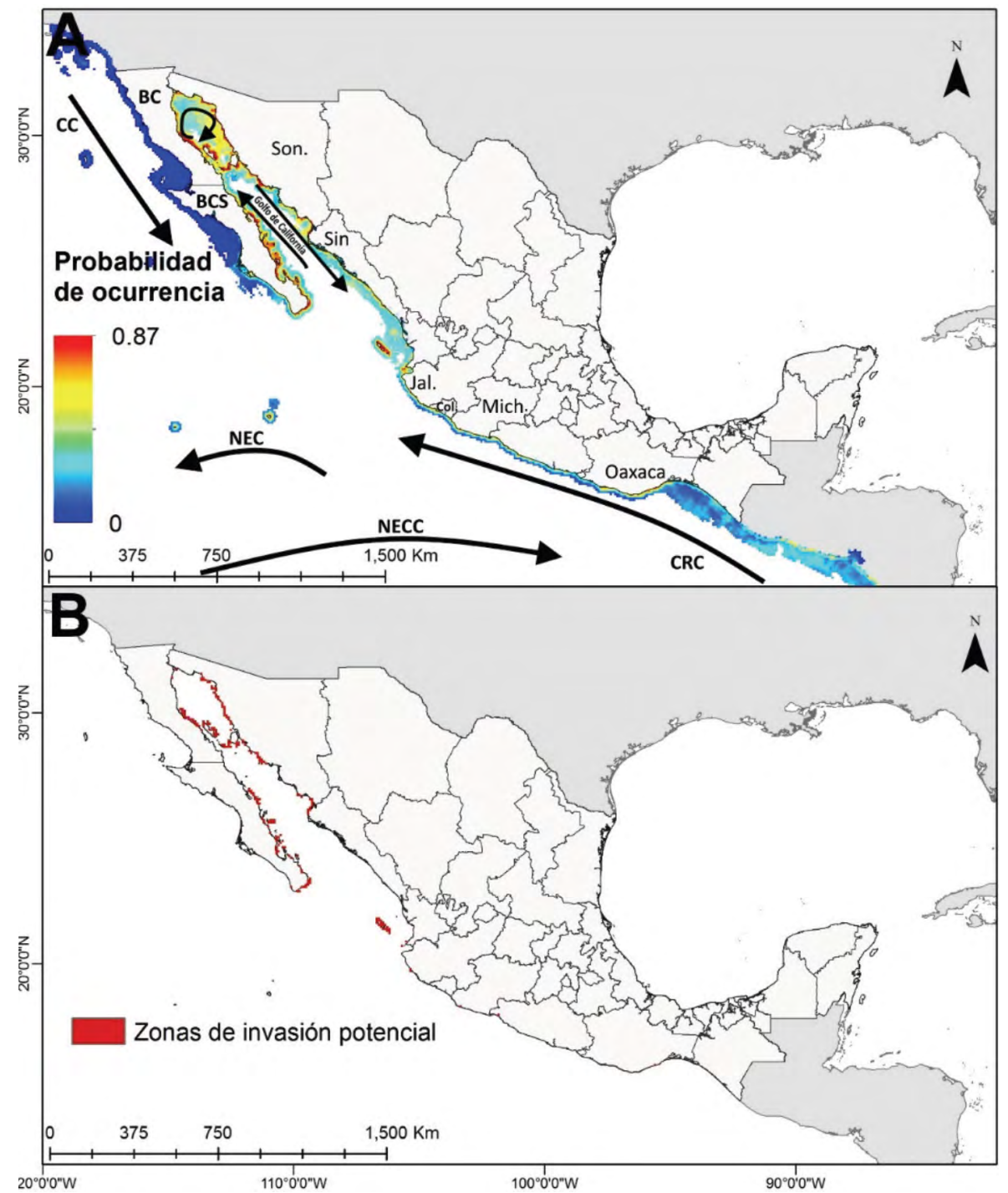

Fig. 1. A) Zonas de invasión potencial del pez león en el Pacífico mexicano, las líneas indican las principales corrientes marinas, las abreviaciones son: Corriente de Costa Rica (CRC), Corriente ecuatorial del norte (NEC), Contracorriente ecuatorial del norte (NECC) y Corriente de California (CC). B) Sitios donde las condiciones serían adecuadas para el establecimiento de poblaciones de pez león

Fig. 1. A) Areas susceptible to lionfish invasion in the Mexican Pacific. Lines indicate the main marine currents. Figure shows Costa Rica Current (CRC), North Equatorial Current (NEC), North Equatorial Counter Current (NECC), and California Current (CC). B) Sites where conditions would be adequate for establishment of lionfish populations 
el algoritmo identifica las condiciones en las que habita el taxón y proyecta el espacio geográfico potencialmente habitable, de acuerdo con las preferencias de hábitat sugeridas por los puntos de ocurrencia.

Para generar el modelo, los datos de presencia de Pterois spp. en el Atlántico occidental y en su región original de distribución (tomados como las fuentes de la posible invasión, de manera análoga al razonamiento de Johnston \& Purkis, 2014), fueron descargados de distintas bases de datos internacionales, como Fishbase (Froese \& Pauly, 2018), Ocean Biogeographic Information System (OBIS: http:// www.iobis.org), Global Biodiversity Information Facility (GBIF: http:// www.gbif.net) y de la base de datos en línea del Servicio Geológico de los Estados Unidos sobre especies acuáticas no indígenas (USGS-NAS: http:// nas.er.usgs.gov). Se eliminaron los registros repetidos entre las diversas bases, y aquellos mal georreferenciados que incluyen puntos en tierra y coordenadas sin grados decimales o notablemente fuera del ámbito latitudinal de ocurrencia del pez, que pudieran representar errores. Posterior a la depuración de datos, se trabajó con un total de 1948 datos para $P$. volitans/ miles en el Indopacífico, así como 5 879 avistamientos individuales en el Atlántico para el periodo 1985-2018.

El área geográfica que abarcó el modelo incluye la totalidad del
Pacífico oriental entre los $42^{\circ} \mathrm{N}$ y $34^{\circ}$ $\mathrm{S}$ (abarca zonas tropicales y subtropicales), y se hizo un recorte desde la línea de costa hasta los $350 \mathrm{~m}$ de profundidad, con base en el intervalo batimétrico en el que habita Pterois spp. (Luiz et al. 2013). Para la caracterización ambiental de dicha región se consideraron los valores promedio, máximo y mínimo en el nivel superficial de temperatura, concentración de clorofila $a$, salinidad y velocidad de la corriente. Se tomó en cuenta además, la temperatura y salinidad en el nivel de fondo marino y finalmente, el tipo de costa (rocoso o arenoso), las cuales influyen en la distribución de Pterois spp. (Kimball et al. 2004; Johnston \& Purkis, 2014; Anton et al. 2014; Valdivia et al. 2014; Jud et al. 2015).

Los datos de temperatura superficial y concentración de clorofila $a$ provienen del sensor MODIS-Aqua para el periodo 2002-2017, a una escala de 5 minutos de arco $(9 \mathrm{~km})$, y en el caso de la salinidad se tomaron del sensor Aquarius (beam 3 v3,0 refined) en la temporada de agosto 2011 a diciembre 2015, y con una resolución espacial de $1^{\circ}$ de latitud-longitud; estos posteriormente fueron interpolados a una escala de 5 minutos geográficos mediante el método de distancia inversa ponderada, con la finalidad de tener una base de datos homogénea con los datos del sensor MODIS-Aqua. Los datos de velocidad máxima, mínima y promedio de la corriente superficial por pixel (m/ 
seg) fueron descargados del proyecto Ocean Surface Current Analyses Real Time de la Administración Oceánica y Atmosférica Nacional de los Estados Unidos (NOAA; /https://podaac. jpl.nasa.gov/) para el periodo 2000 a 2015, e interpolados a $9 \mathrm{~km}$.

Además de la información satelital, los registros ambientales fueron complementados con datos de temperatura y salinidad del World Ocean Atlas 2013 en el nivel del fondo marino, que originalmente estaban a una resolución de $0.25^{\circ}$ y fueron interpolados a 5 minutos geográficos. Para determinar el tipo de costa, se utilizó el índice de vegetación normalizado (NDVI, por sus siglas en inglés), los datos fueron descargados de Global Land Cover Facility (GLCF: http://www.landcover.org/), con él es posible diferenciar entre ecosistemas de manglar y otro tipo de vegetación no sumergida (Euán-Avila \& Cuevas-Jiménez, 2010), además de potenciar la diferenciación de sitios donde está ausente, como la costa rocosa o playas arenosas, sitios en los cuales los valores de NDVI son muy bajos.

Para conocer los sitios potencialmente habitables por $P$. volitans/ miles en el Pacífico mexicano, se empleó el programa MaxEnt utilizando el $25 \%$ de los datos de ocurrencia para la evaluación del desempeño del algoritmo como sugiere Franklin (2010). La eficiencia de los modelos se estimó al emplear el área bajo la curva (AUC) del gráfico de características operativas del receptor (ROC; receiver operating characteristics), que considera valores superiores a 0.80 como indicadores de buen desempeño (Elith et al. 2011). Se realizaron 30 réplicas con el $75 \%$ de los datos de ocurrencias tomados de manera aleatoria para el entrenamiento y se tomó el promedio de los 30 rásters resultantes como el resultado de la modelación del nicho ecológico y la distribución potencial de Pterois spp. (Stranges et al. 2019). Se seleccionó salida logística, por lo que los valores del ráster de salida del modelo van de 0 a 1 , donde valores cercanos a 1 indican mejores condiciones de hábitat para el establecimiento de la especie.

Una vez creado el modelo de distribución geográfica potencial, se utilizó el parámetro de $10 \%$ percentil proporcionado por MaxEnt para generar un mapa binario (presencia/ ausencia; Melo-Merino et al. 2020) en el que, todos los sitios en el ráster de distribución potencial con valores superiores a este umbral se consideran óptimos para el establecimiento de la especie, ya que cuenta con características ambientales idóneas para su presencia ante una invasión potencial, mientras que valores inferiores a él se asumen como sitios no óptimos, por lo que se esperaría su ausencia.

Una vez detectados los sitios adecuados para la presencia de Pterois spp., para hacer estimaciones del impacto que tendría su llegada sobre la biodiversidad 
del Pacífico mexicano, se realizó una intersección espacial entre los sitios óptimos para la invasión y los mapas de ámbito geográfico ocupado por peces con preferencias arrecifales y tallas menores a $13 \mathrm{~cm}$, que son sus principales presas (Green et al. 2014); los datos se tomaron de la página web de Peces Costeros del Pacífico Oriental Tropical (Robertson \& Allen, 2018). Adicionalmente, en otro análisis se incluyeron especies de mayor tamaño y de interés comercial que pueden ser presas de Pterois spp. en estadios juveniles, y para ello, se realizaron intersecciones espaciales de sus zonas de captura (presentadas por Ulloa et al., 2006) y los sitios potenciales de colonización de $P$. volitans/miles.

\section{RESULTADOS}

Con los puntos de ocurrencia y las variables ambientales previamente descritas, los análisis en MaxEnt indican que el área bajo la curva del modelo de nicho ecológico y distribución potencial de Pterois spp. fue de 0.856 \pm 0.004 (promedio y error típico). La salinidad en el nivel del fondo marino, el tipo de costa (rocoso) y la temperatura superficial promedio fueron los factores clave para predecir las ocurrencias, y sumaron más del $65 \%$ de la contribución al modelo. Los demás factores ambientales tuvieron un aporte estadísticamente menor (Cuadro 1).

Cuadro 1. Porcentaje de contribución de las variables ambientales utilizadas en el modelo MaxEnt para el pez león (Pterois volitans/miles)

Table 1. Contribution percent of environmental variables used in the MaxEnt model for lionfish (Pterois volitans/miles)

\begin{tabular}{lcc}
\hline Variable & $\begin{array}{c}\text { Porcentaje de } \\
\text { contribución (\%) }\end{array}$ & $\begin{array}{c}\text { Importancia de } \\
\text { permutación }\end{array}$ \\
\hline Salinidad del fondo & 28.5 & 12.8 \\
Tipo de costa & 18.9 & 22.8 \\
Temperatura promedio & 17.9 & 15.1 \\
Salinidad promedio & 8.7 & 15.8 \\
Temperatura máxima & 5.6 & 6.0 \\
Temperatura mínima & 5.3 & 11.7 \\
Clorofila promedio & 2.6 & 1.4 \\
Salinidad mínima & 2.5 & 4.3 \\
Temperatura del fondo & 2.3 & 1.7 \\
Salinidad máxima & 1.9 & 1.8 \\
Velocidad de corriente máxima & 1.9 & 1.7 \\
Clorofila mínima & 1.6 & 1.4 \\
Clorofila máxima & 1.4 & 2.1 \\
Velocidad de corriente promedio & 0.6 & 1.0 \\
Velocidad de corriente mínima & 0.4 & 0.3 \\
\hline
\end{tabular}


De acuerdo con el mapa de distribución potencial obtenido (Figs. 1A, 1B), en la parte sur del Pacífico mexicano existen pocas localidades donde el entorno es apto para el asentamiento de Pterois spp., y se circunscriben a sitios puntuales en Oaxaca, Colima y Michoacán (latitudes $16^{\circ}$ a $18^{\circ} \mathrm{N}$ ), en contraste, las costas de Jalisco y en específico, en bahía de Banderas, isla Isabel y las islas Marías presentan buenas condiciones para su establecimiento. Por otra parte, la probabilidad de ocurrencia de la especie, dado el caso de su introducción, sería más alta $\mathrm{y}$ espacialmente casi continua en el centro y norte del GC, aunque también hay sitios aptos de colonización al norte de Sinaloa, el sur de Sonora (ambas en $\left.27^{\circ} \mathrm{N}\right)$ y toda la costa oriental de Baja California Sur, desde $\operatorname{los} 23^{\circ} \mathrm{N}$ a $\operatorname{los} 28^{\circ} \mathrm{N}$ (Fig. 1A). Los mapas también indican que las islas oceánicas del oeste de México (Revillagigedo, Alijos, Guadalupe) no parecen presentar condiciones adecuadas para una potencial invasión del pez. Finalmente, la probabilidad de ocurrencia de Pterois spp. es muy baja al oeste de la península de Baja California, por lo que la región de cabo San Lucas $\left(23^{\circ} \mathrm{N}\right)$ puede ser definida como el límite para la zona apta de ocurrencia del pez invasor.

De acuerdo con el análisis espacial, se encontró que la posible presencia de Pterois spp. en el Pacífico mexicano coincidiría geográficamente con la distribución de 198 especies de peces de talla pequeña $(\leq 13 \mathrm{~cm})$. Las especies encontradas pertenecen a 13 órdenes, 35 familias y 88 géneros. Las familias en mayor riesgo ecológico derivado de una invasión de Pterois spp. son Gobiidae (46 especies), Labrisomidae (27 especies) y Chaenopsidae (16 especies). Entre las zonas donde más especies se verían afectadas (Fig. 2A) destacan las islas Tiburón y Ángel de la Guarda (29 $\mathrm{N})$, se incluyen sitios cercanos a la bahía de Guaymas $\left(28^{\circ} \mathrm{N}\right)$. Además, desde cabo San Lucas hasta Loreto $\left(25^{\circ} \mathrm{N}\right)$ en Baja California, y en las islas Marías y bahía de Banderas en la boca del GC.

Por otra parte, el análisis espacial encontró que 31 pesquerías del GC se desarrollan en sitios con potencial para la llegada del pez león, siendo la región entre el norte de Sinaloa hasta isla Tiburón, en latitudes 28 a $32^{\circ} \mathrm{N}$ (Fig. 2B) la que podría verse más afectada, debido a que acumula hasta 18 distintas zonas de captura. En la costa oeste del golfo, las secciones costeras más susceptibles se encuentran desde Loreto $\left(25^{\circ} \mathrm{N}\right)$ hasta el norte de BCS $\left(28^{\circ} \mathrm{N}\right)$, además del sureste de esa entidad, con hasta 13 pesquerías amenazadas, entre las que se encuentran las de camarones, jaibas y moluscos bivalvos, además de grupos de peces como pargos, meros, pericos, entre otros. 

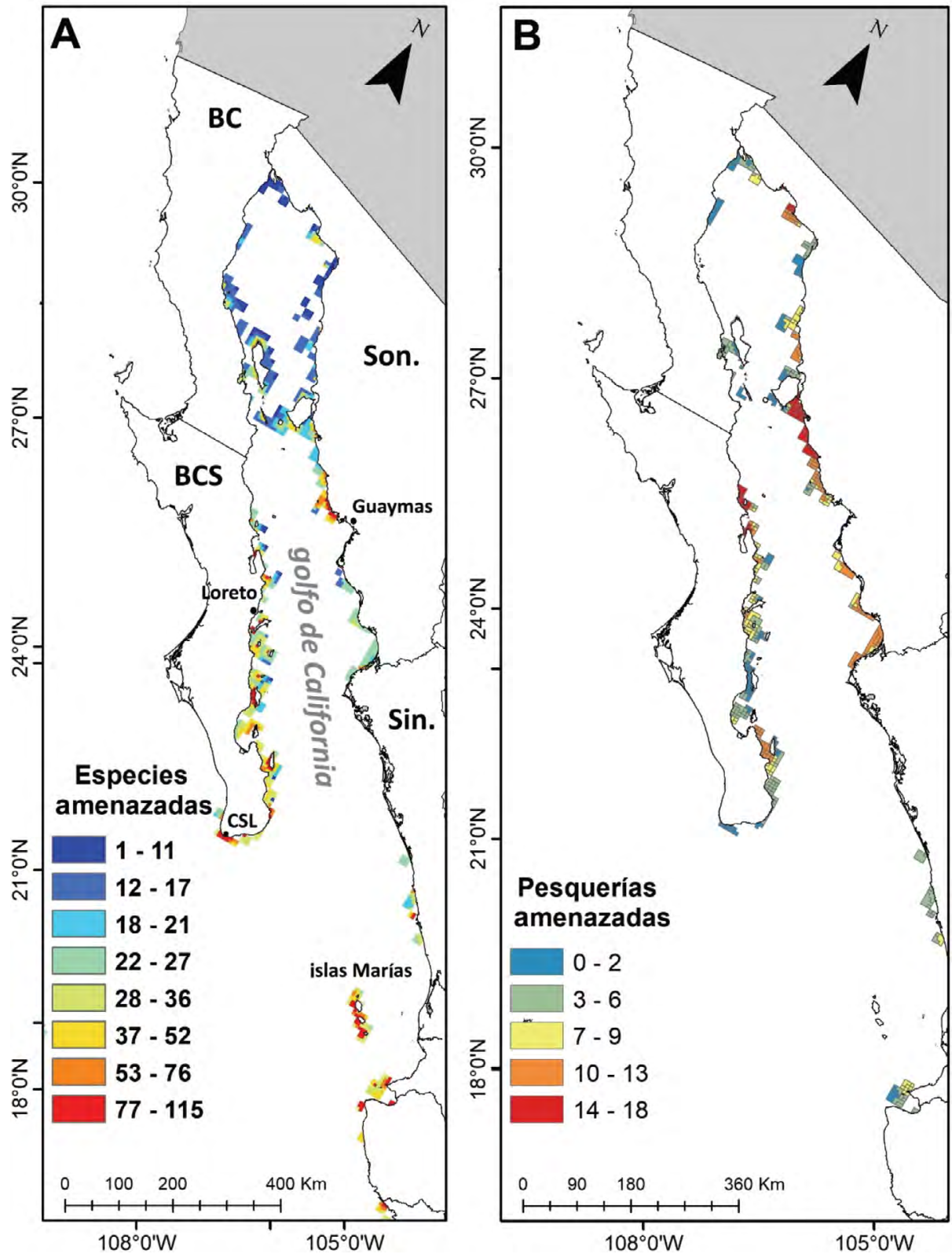

Fig. 2. A) Número de especies potencialmente amenazadas en el golfo de California ante una posible invasión del pez león (Pterois volitans/miles). B) Número de pesquerías que podrían verse afectadas por la invasión del pez león

Fig. 2. Number of potentially threatened species in the Gulf of California before a possible lionfish (Pterois volitans/miles) invasion. B) Number of fishing zones that would be impacted by lionfish invasion 


\section{DISCUSIÓN}

Los análisis de nicho ecológico y distribución potencial de taxas están siendo cada vez más utilizados por la comunidad científica, y en el caso de organismos marinos se emplean con frecuencia para determinar patrones espaciales del hábitat de grupos de importancia comercial o ecológica, sitios de ocurrencia de especies invasoras, y proyecciones de cambio climático (Melo-Merino et al. 2020). Adicionalmente se han establecido como esenciales para investigaciones en campos relacionados con la ecología, el manejo y la conservación (Peterson \& Anamza, 2015; Melo-Merino et al. 2020). No obstante, aún presentan una serie de inconvenientes relacionados a la disponibilidad de variables ambientales a la escala correcta de modelado (Connor et al. 2017), y en los algoritmos predictivos aún no es posible incorporar datos sobre variables ligadas a las interacciones ecológicas o el comportamiento de los organismos (Anderson, 2017).

Los problemas citados también se presentaron en este estudio, dando inicio por la limitada disponibilidad de variables ambientales obtenidas de manera original a una escala tan fina como la empleada (9 $\mathrm{km}$ de pixel), ya que los datos sobre factores como nutrientes, concentración de oxígeno y muchos otros, solo se pueden generar de manera directa en cruceros oceanográficos, que, por lo general, se conducen sobre aguas profundas. La consecuencia de ello es que se requiere interpolar datos si se quiere tener información de la zona costera (Tyberghein et al. 2012), y con ello se pueden cometer errores en la precisión que afecten los resultados (Robinson et al. 2017).

En el presente estudio, los pronósticos de zona posible de ocurrencia del pez león se basaron en datos sobre cinco variables ambientales obtenidos por medio de sensores remotos a la resolución espacial adecuada, y estos fueron complementados con registros in situ del promedio de temperatura y salinidad en el fondo marino. Los resultados demostraron que ese enfoque ofrece un acercamiento adecuado para juzgar el potencial de colonización de Pterois spp. en el Pacífico mexicano ya que se obtuvo un AUC $>0.8$ (que indica buen desempeño; Elith, 2000) y, además el presente estudio permite avanzar el conocimiento, ya que el trabajo previo sobre el tema en el Pacífico oriental (Johnston \& Purkis, 2014) empleó solamente tres variables (profundidad, promedio de la temperatura y las corrientes marinas) y a una escala geográfica comparativamente mucho más baja.

De acuerdo con el programa MaxEnt, las variables con mayor influencia en la distribución de Pterois spp. fueron la salinidad del fondo, 
tipo de costa y temperatura promedio (Cuadro 1). La relevancia del primer elemento se explica porque el pez león tiene hábitos demersales, en donde la salinidad puede tener poca variación si se compara con la superficie, donde hay influencia de agua dulce proveniente de lluvias y descarga de ríos. La preferencia de Pterois spp. al tipo de costa podría asociarse a que arrecifes coralinos o rocosos pueden concentrar mayor disponibilidad de presas (básicamente peces pequeños). La tercera variable más importante fue la temperatura promedio, la cual es clave en la distribución de casi todos los organismos (Melo-Merino et al. 2020). El pez león tiene amplia tolerancia térmica (13-32 ${ }^{\circ}$ C; Dabruzzi et al. 2017), no obstante, se restringe a zonas tropicales y subtropicales, lo cual es consistente con los resultados encontrados en este estudio (Fig. 1B).

La velocidad de las corrientes marinas no influyó en el modelo debido a que este estudio consideró, en exclusivo, la intensidad de las corrientes, no la movilidad potencial de las larvas. Sin embargo, el factor no puede dejarse de lado al considerar la posibilidad de dispersión de la especie en el Pacífico mexicano (Fig. 1A). Johnston y Purkis (2014) concluyen que esta región es vulnerable ante una invasión de Pterois spp. originada en el sur, debido a que las principales corrientes del Pacífico oriental tropical fluyen con dirección norte, y mantienen alta conectividad a lo largo de la región (Montaño-Cortés \& Marinone, 2016). Por otro lado, el potencial de éxito de establecimiento de Pterois spp., como el de cualquier otra especie, tiene limitantes relacionadas a aspectos biológicos como la competencia y la depredación (Johnston \& Purkis, 2014), los cuales aún no pueden ser incluidos de forma confiable en los modelos tipo MaxEnt (Anderson, 2017), pero que complementarían de excelente forma este tipo de estudios (Melo-Merino et al. 2020).

A partir del mapa de distribución potencial pueden conocerse las zonas potenciales de éxito de asentamiento de Pterois spp., y con ello, realizar un examen de las afectaciones potenciales que puede causar la especie donde la susceptibilidad de invasión fuese mayor. En este caso, los resultados (Fig. 1B) señalan de manera clara que el GC y zonas adyacentes tienen las condiciones oceanográficas más aptas para el establecimiento de Pterois spp., lo cual coincide con las afirmaciones de MacIsaac et al. (2016) al revisar el potencial de inmigración de Pterois spp. hacia la costa occidental del continente americano por medio de técnicas estadísticas no lineales y registros que evidencian la posibilidad de llegada de la especie al Pacífico americano dentro de agua de lastre liberada por embarcaciones mayores que cruzan el canal de Panamá desde el Atlántico, o vienen del Pacífico oeste. Johnston y Purkis (2014) también señalaron que la región 
de bahía de Banderas a Nayarit $\left(20^{\circ} \mathrm{N}\right.$ a $22^{\circ} \mathrm{N}$, en la costa oriental del GC) era la que tenía el mayor potencial para reclutamiento larval de Pterois spp.

En resumen, el conjunto de los trabajos citados más los resultados aquí presentados, advierten que, si $P$. volitans/miles llega a ser introducido en el occidente de las Américas, el GC tendría altas posibilidades de ser colonizado. Esto se debe a sus características oceanográficas, pues cuenta con alta salinidad y temperaturas que van de $16^{\circ}$ a $32^{\circ} \mathrm{C}$ en la parte central (Soto-Mardones et al. 1999), lo cual se encuentra dentro de la tolerancia térmica de la especie (Dabruzzi et al. 2017), además de gran disponibilidad de arrecifes rocosos con alta diversidad y abundancia de peces, particularmente en la costa oeste del GC y en Sonora (Thomson et al. 2000; TurkBoyer et al. 2014).

Además del mapa de zonas potenciales de invasión por Pterois spp., se consideró importante hacer un análisis de los efectos ecológicos potenciales de la llegada de la especie al GC. Dado que esa especie consume, principalmente, presas de tamaños de $13 \mathrm{~cm}$ o menos de longitud total (Green et al. 2014), revisando los registros sobre ámbito geográfico de dichos taxa (Robertson y Allen, 2018) se encontró que existen 198 especies como presas potenciales incluyendo 28 endémicas del GC (familia Gobiidae con 14 especies, Labrisomidae con
6 especies y Chaenopsidae con 8 especies), Todas ellas, debido a su limitada distribución geográfica (Morzaria-Luna et al. 2018), podrían ser afectadas de manera relevante por la llegada del pez león, viéndose afectado su reclutamiento, como ha ocurrido con algunas especies en el Atlántico (Albins \& Hixon 2008; Ballew et al. 2016). Cabe señalar que muchos puntos de alta probabilidad de aparición de Pterois spp. también corresponden a las zonas de mayor biodiversidad de macroinvertebrados del GC (Ulate et al. 2016), los cuales también podrían pasar a formar parte de la dieta de Pterois spp.

Otro aspecto relevante del GC son sus niveles de producción pesquera, ya que este mar interior aporta el $75 \%$ de la captura total en México (Arreguín-Sánchez et al. 2017). En ese sentido, destacan los camarones azul, blanco y café (Farfantepenaeus californiensis, Litopenaeus vannamei, L. stylirostris), cuya pesquería representa el mayor beneficio económico en la región (Lluch-Cota et al. 2007). Estas especies habitan principalmente en la costa oriental del GC, en zonas arenosas cercanas a los sitios donde de acuerdo al análisis, Pterois spp. podría establecerse. Si es el caso y al considerar que los camarones representan una fuente de alimento importante para este depredador en sus sitios originales de residencia (Layman et al. 2014; Peake et al. 2018), sería factible que Pterois spp. pudiese representar un problema 
para una pesquería fundamental para el bienestar de miles de personas.

Adicionalmente, el pez invasor podría afectar también la producción de jaiba verde (Callinectes bellicosus), y disminuir el éxito de algunas especies de peces de importancia comercial como cabrillas, meros y peces perico al consumir sus juveniles en los arrecifes rocosos (Ulloa et al. 2006). En particular y de acuerdo con nuestros resultados, en la costa de la península de Baja California existen condiciones óptimas para la colonización de Pterois spp. (Fig. 2B), lo que podría afectar el desempeño del refugio pesquero San Cosme-Punta Coyote (Espinosa-Romero et al. 2014), localizado al sur de Loreto $\left(24^{\circ} \mathrm{N}\right)$, y uno de los más importantes de México (Villaseñor-Derbez et al., 2019). Por otra parte, en la costa continental, el estado de Sonora podría ser la entidad más afectada, al concentrar el mayor número de pesquerías en sitios óptimos de colonización, además de ser una de las entidades que genera mayor volumen de pesca a escala nacional y el pez podría también afectar el funcionamiento del corredor pesquero Puerto Lobos - Puerto Peñasco, Sonora (Turk-Boyer et al. 2014). Por todo lo anterior, se puede considerar que la llegada de Pterois spp. al Pacífico mexicano, en particular al GC, representaría no solo un problema ecológico, sino que su llegada podría tener graves consecuencias económicas, que afectan a las principales industrias pesqueras locales.

\section{CONCLUSIONES}

Los resultados del modelo de nicho ecológico y distribución espacial aplicado, mostraron que en la parte central de la costa oeste de México y en el occidente de la península de Baja California, las condiciones oceanográficas no parecen ser adecuadas para la colonización exitosa de Pterois spp.; en contraste, en el GC esta especie bien podría establecerse si existieran los agentes que permitieran su llegada. La llegada de este pez nocivo a este mar interior podría afectar a casi 200 especies de peces e invertebrados que ocupan hábitats arrecifales y tienen la talla idónea para ser objeto de depredación, entre ellas casi 30 endémicas y de importancia comercial, por lo que puede afirmarse que la entrada del pez león tendría un impacto sensible sobre las actividades económicas y la salud de uno de los centros de biodiversidad marina del planeta

\section{AGRADECIMIENTOS}

Se agradecen los comentarios de Gabriela Cruz (UABCS), Sara Melo (Instituto de Ciencias del Mar y Limnología, UNAM) y Magdalena Précoma (Comunidad y Biodiversidad, A.C.) a versiones preliminares 
del documento y a los revisores anónimos que enriquecieron el contenido de este trabajo.

\section{REFERENCIAS}

Albins, M. A. \& Hixon, M. A. (2008). Invasive Indo-Pacific lionfish Pterois volitans reduce recruitment of Atlantic coral-reef fishes. Mar. Ecol. Prog. Ser., 367, 233-238.

Anderson, R. P. (2017). When and how should biotic interactions be considered in models of species niches and distributions? J Biogeogr., 44(1), 8-17. https:// doi.org/10.1111/jbi.12825

Anton, A., Simpson, M. S. \& Vu, I., (2014). Environmental and biotic correlates to lionfish invasion success in Bahamian coral reefs. PloS One, 9(9), e106229. https://doi.org/10.1371/journal. pone.0106229

Arreguín-Sánchez, F., del Monte-Luna, P., Zetina-Rejón, M. J. \& Albáñez-Lucero, M. O. (2017). The Gulf of California large marine ecosystem: Fisheries and other natural resources. Environ. Dev., 22, 71-77.

Azzurro, E., Soto, S., Garofalo, G. \& Maynou, F. (2013). Fistularia commersonii in the Mediterranean Sea: invasion history and distribution modeling based on presence-only records. Biol. Invasions, 15(5), 977-990.

Ballew, N. G., Bacheler, N. M., Kellison, G. T. \& Schueller, A. M. (2016). Invasive lionfish reduce native fish abundance on a regional scale. Scien. Rep., 6, 32169.

Bariche, M., Torres, M. \& Azzurro, E. (2013). The presence of the invasive lionfish Pterois miles in the Mediterranean Sea. Mediterr. Mar. Sci., 14(2), 292-294. http://dx.doi.org/10.12681/mms.428
Bernal, N. A., DeAngelis, D. L., Schofield, P. J. \& Sealey, K. S. (2014). Predicting spatial and temporal distribution of Indo-Pacific lionfish (Pterois volitans) in Biscayne Bay through habitat suitability modeling. Biol. Invasions, 17(6), 1603-1614.

Connor, T., Hull, V., Viña, A., Shortridge, A., Tang, Y., Zhang J. ... \& Liu, J. (2017). Effects of grain size and niche breadth on species distribution modeling. Ecography, 41(8), 1270-1282. https://doi. org/10.1111/ecog.03416

Crocetta, F., Agius, D., Balistreri, P., Bariche, M., Bayhan, Y. K., Çakir, M. ... \& Ergüden, D. (2015). New Mediterranean biodiversity records (October 2015). Mediterr. Mar. Sci., 16(3), 682-702. http://dx.doi.org/10.12681/ mms. 1477

Dabruzzi, T. F., Bennett, W. A. \& Fangue, N. A. (2017). Thermal ecology of red lionfish Pterois volitans from southeast Sulawesi, Indonesia, with comparisons to other Scorpaenidae. Aquat. Biol., 26, 1-14.

Elith, J. (2000). Quantitative methods for modeling species habitat: comparative performance and an application to Australian plants. In S. Ferson \& M. Burgman (Eds.), Quantitative methods for conservation biology (pp. 39-58). EE. UU.: Springer-Verlag. https://doi. org/10.1007/0-387-22648-6 4

Elith, J., Phillips, S. J., Hastie, T., Dudik, M., Chee, Y. E. \& Yates C. J. (2011). A statistical explanation of MaxEnt for ecologists. Divers. Distrib., 17(1), 43-57. https://doi. org/10.1111/j.1472-4642.2010.00725.x

Espinosa-Romero, M. J., Rodríguez, L. F., Weaver, A. H., Villanueva-Aznar, C., \& Torre, J. (2014). The changing role of NGOs in Mexican small-scale fisheries: From environmental conservation 
to multi-scale governance. Marine Policy, 50, 290-299.

Euán-Avila, J. I. \& Cuevas-Jiménez, A. (2010). Regionalizing coastal zones with geospatial tools for integrated coastal zone management. In D. R. Green (Ed.), Coastal and marine geospatial technologies (pp. 139-151). Netherlands: Springer Press.

Ferreira, C. E. L., Luiz, O. J., Floeter, S. R., Lucena, M. B., Barbosa, M. C., Rocha, C. R., Rocha \& L. A. (2015). First record of invasive lionfish (Pterois volitans) for the Brazilian Coast. PLoS ONE, 10(4), e0123002. https://doi. org/10.1371/journal.pone.0123002

Fiedler, P. C. \& Talley, L. D. (2006). Hydrography of the eastern tropical Pacific: a review. Prog. Oceanogr., 6(2-4), 143-180. https://doi.org/10.1016/j. pocean.2006.03.008

Franklin, J. (2010). Mapping species distributions: spatial inference and prediction. U. K.: Cambridge University Press.

Froese, R. \& Pauly, D. (2018). Fishbase. World Wide Web electronic publication. 2017. http://www. Fishbase.org. Acceso: septiembre 2018.

García-Molinos, J., Halpern, B. S., Schoeman, D. S., Brown, C. J., Kiessling, W., Moore, P. J. ... \& Burrows, M. T. (2016). Climate velocity and the future global redistribution of marine biodiversity. Nat. Clim. Change, 6(1), 83-88. https:// doi.org/10.1038/nclimate2769

Gaston, K. J. (2003). The structure and dynamics of geographic ranges. U. K.: Oxford University Press.

Glynn, P. W., Alvarado, J. J., Banks, S. Cortés, J., Feingold, J. S., Jiménez, C. ... \& Navarrete, S. (2017). Eastern Pacific coral reef provinces, coral community structure and composition: an overview. In P. W. Glynn, D. P. Manzello \& I. C. Enochs (Eds.), Coral reefs of the eastern tropical Pacific (pp. 107-176). Netherlands: Springer Press.

Green, S. J., Dulvy, N. K., Brooks, A. M., Akins, J. L., Cooper, A. B., Miller, S. \& Côté, I. M. (2014). Linking removal targets to the ecological effects of invaders: a predictive model and field test. Ecol. Appl., 24(6), 1311-1322. https:// doi.org/10.1890/13-0979.1

Johnston, M. W. \& Purkis, S. J. (2011). Spatial analysis of the invasion of lionfish in the western Atlantic and Caribbean. Mar. Pollut. Bull., 62(6), 1218-1226. https://doi.org/10.1016/j. marpolbul.2011.03.028

Johnston, M. W. \& Purkis, S. J. (2012). Invasionsoft: a web-enabled tool for invasive species colonization predictions. Aquat. Invasions, 7(3), 405-417. https://doi.org/10.3391/ai.2012.7.3.011

Johnston, M. W. \& Purkis, S. J. (2014). Lionfish in the eastern Pacific: a cellular automaton approach to assessing invasion risk. Biol. Invasions, 16(12), 2681-2695. https://doi.org/10.1007/ s10530-014-0696-Z

Jud, Z. R., Nichols, P. K. \& Layman, C. A. (2015). Broad salinity tolerance in the invasive lionfish Pterois spp. may facilitate estuarine colonization. Environ. Biol. Fish., 98(1), 135-143. https://doi. org/10.1007/s10641-014-0242-y

Kimball, M. E., Miller, J. M., Whitfield, P. E. \& Hare J. A. (2004). Thermal tolerance and potential distribution of invasive lionfish (Pterois volitans/miles complex) on the east coast of the United States. Mar. Ecol. Prog. Ser., 283, 269-278. https://doi.org/10.3354/ meps 283269

Kletou, D., Hall-Spencer, J. M. \& Kleitou, P. (2016). A lionfish (Pterois miles) invasion has begun in the Mediterranean Sea. Mar. Biodivers. Rec., 
9(1), 46. https://doi.org/10.1186/ s41200-016-0065-y

Layman, C. A., Jud, Z. R. \& Nichols, P. (2014). Lionfish alter benthic invertebrate assemblages in patch habitats of a subtropical estuary. Mar. Biol., 161(9), 2179-2182.

Leidenberger, S., Obst, M., Kulawik, R. Stelzer, K., Heyer, K., Hardisty, A. ... \& Bourlat, S. J. (2015). Evaluating the potential of ecological niche modelling as a component in marine non-indigenous species risk assessments. Mar. Pollut. Bull., 97(1-2), 470-487. https://doi.org/10.1016/j.marpolbul.2015.04.033

Lessios, H. A. \& Baums, I. B. (2017). Gene flow in coral reef organisms of the Tropical Eastern Pacific. In P. W. Glynn, D. P. Manzello \& I. C. Enochs (Eds.), Coral reefs of the Eastern Tropical Pacific (pp. 477-500). Netherlands: Springer Press.

Lluch-Cota, S. E., Aragon-Noriega, E. A., Arreguín-Sánchez, Aurioles-Gamboa, D., Bautista-Romero, J. J., Brusca, R. C. ... \& Fernández, G. (2007). The Gulf of California: Review of ecosystem status and sustainability challenges. Prog. Oceanogr., 73(1), 1-26.

Luiz, O. J., Floeter, S. R., Rocha, L. A. \& Ferreira, C. E. (2013). Perspectives for the lionfish invasion in the South Atlantic: are Brazilian reefs protected by the currents. Mar. Ecol. Prog. Ser., 485, 1-7. https://doi.org/10.3354/meps10383

MacIsaac, H. J., De Roy, E. M., Leung, B., Grgicak-Mannion, A. \& Ruiz, G. M. (2016). Possible ballast water transfer of Lionfish to the eastern Pacific Ocean. PLoS ONE, 11(11), e0165584. https:// doi.org/10.1371/journal.pone.0165584

Melo-Merino, S. M., Reyes-Bonilla, H. \& Lira-Noriega, A. (2020). Ecological niche models and species distribution models in marine environments: A literature review and spatial analysis of evidence. Ecol. Model., 415, 108837. https://doi. org/10.1016/j.ecolmodel.2019.108837

Montaño-Cortés, C. \& Marinone, S. G. (2016). Three-dimensional connectivity during summer in the northern Gulf of California. Sci. Mar., 80(3), 409-421. https:// doi.org/10.3989/scimar.04370.15A

Morzaria-Luna, H. N., Cruz-Piñón, G., Brusca, R. C., López-Ortiz, A. M., Moreno-Báez, M., Reyes-Bonilla, H. \& Turk-Boyer, P. (2018). Biodiversity hotspots are not congruent with conservation areas in the Gulf of California. Biod. Cons., 27(14), 3819-3842.

Peake, J., Bogdanoff, A. K., Layman, C. A., Castillo, B., Reale-Munroe, K., Chapman, J. ... \& Faletti, M. (2018). Feeding ecology of invasive lionfish (Pterois volitans and Pterois miles) in the temperate and tropical western Atlantic. Biol. Invasions, 20(9), 2567-2597.

Peterson, A. T. \& Anamza, T. (2015). Ecological niches and present and historical geographic distributions of species: a 15-year review of frameworks, results, pitfalls, and promises. Folia Zool., 64(3), 207-217. https://doi. org/10.25225/fozo.v64.i3.a3.2015

Peterson, A. T., Soberón, J., Pearson, R. G., Anderson, R., Martínez-Meyer, E., Nakamura M. \& Araújo, M. B. (2011). Ecological niches and geographic distributions. EE. UU.: Princeton University Press.

Phillips, S. J., Anderson, R. P. \& Schapire, R. E. (2006). Maximum entropy modeling of species geographic distributions. Ecol. Model., 190(3-4), 231-259. https://doi. org/10.1016/j.ecolmodel.2005.03.026

Robertson, D. R. \& Allen, G. R. (2018). Peces costeros del Pacífico Oriental Tropical: sistema de Información en línea. Versión 2.0 Instituto Smithsoniano de Investigaciones Tropicales, 
Balboa, República de Panamá. disponible en http://biogeodb.stri.si.edu/ sftep/es/pages.

Robinson, N. M., Nelson, W. A., Costello, M. J., Sutherland, J. E. \& Lundquist, C. J. (2017). A systematic review of marine-based species distribution models (SDMs), with recommendations for best practice. Front. Mar. Sci., 18(4), 1-11. https://doi.org/10.3389/ fmars.2017.00421

Santander-Monsalvo, J., López-Huerta, I., Aguilar-Perera, A. \& Tuz-Sulub, A. (2012). First record of the red lionfish (Pterois volitans [Linnaeus, 1758]) off the coast of Veracruz, Mexico. BioInvasions Rec., 1(2), 121-124. https://doi. org/10.3391/bir.2012.1.2.07

Schofield, P. J. (2009). Geographic extent and chronology of the invasion of non-native lionfish (Pterois volitans [Linnaeus 1758] and P. miles [Bennett 1828]) in the Western North Atlantic and Caribbean Sea. Aquat. Invasions, 4(3), 473-474. https://doi.org/10.3391/ ai.2009.4.3.5

Schofield, P. J. (2010). Update on geographic spread of invasive lionfishes (Pterois volitans [Linnaeus 1758] and P. miles [Bennett 1828]) in the Western North Atlantic Ocean, Caribbean Sea and Gulf of Mexico. Aquat. Invasions, 5(Supplement 1), S117-S122. https:// doi.org/10.3391/ai.2010.5.S1.024

Schofield, P. J., Huge, D. H., Rezek, T. C., Slone, D. H. \& Morris, J. A. (2015). Survival and growth of invasive Indo-Pacific lionfish at low salinities. Aquat. Invasions, 10, 333-337.

Stranges, S., Cuervo-Robayo, A. P., Martínez-Meyer, E., Morzaria-Luna, H. N. \& Reyes-Bonilla, H. (2019). Distribución potencial bajo escenarios de cambio climático de corales del género Pocillopora (Anthozoa: Scleractinia) en el Pacífico oriental tropical. Rev. Mex. Biodivers., 90, e902696.

Turk-Boyer P., Morzaria-Luna H., Martinez-Tovar I., Downton-Hoffmann C. \& Munguia-Vega A. (2014). Ecosystem-Based Fisheries Management of a Biological Corridor Along the Northern Sonora Coastline (NE Gulf of California). In F. Amezcua, B. Bellgraph (Eds) Fisheries Management of Mexican and Central American Estuaries. Estuaries of the World (pp. 125154). Dordrecht., Springer.

Thomson, D. A., Findley, L. T. \& Kerstitch, A. N. (2000). Reeffishes of the Sea of Cortez: the rocky-shore fishes of the Gulf of California. EE. UU.: University of Texas Press.

Tyberghein, L., Verbruggen, H., Pauly, K., Troupin, C., Mineur, F. \& De Clerck, O. (2012). Bio-ORACLE: a global environmental dataset for marine species distribution modelling. Global Ecol. Biogeogr., 21(2), 272281. https://doi.org/10.1111/j.14668238.2011.00656.x

Ulate, K., Sánchez, C., Sánchez-Rodríguez, A., Alonso, D., Aburto-Oropeza, O., \& Huato-Soberanis, L. (2016). Latitudinal regionalization of epibenthic macroinvertebrate communities on rocky reefs in the Gulf of California. Marine Biology Research, 12(4), 389-401.

Ulloa, R., Torre, J., Bourillón, L., Gondor, A. \& Alcantar, N. (2006). Planeación ecorregional para la conservación marina: Golfo de California y costa occidental de Baja California Sur. México. Comunidad y Biodiversidad, A. C.

Valdivia, A., Bruno, J. F., Cox, C. E., Hackerott, S. \& Green, S. J. (2014). Re-examining the relationship between invasive lionfish and native grouper in the Caribbean. PeerJ, 2, e348. https://doi.org/10.7717/ peerj.348 
Villaseñor-Derbez, J. C., Aceves-Bueno, E., Fulton, S., Suarez, A., Hernandez-Velasco, A., Torre, J., \& Micheli, F. (2019). An interdisciplinary evaluation of community-based TURF-reserves. PloS One, 14(8), e0221660. https://doi. org/10.1371/journal.pone.0221660

Whitfield, P. E., Gardner, T., Vives, S. P., Gilligan, M. R., Courtenay Jr, W. R., Ray, G. C. \& Hare, J. A. (2002). Biological invasion of the Indo-Pacific lionfish
Pterois volitans along the Atlantic coast of North America. Mar. Ecol. Prog. Ser., 235, 289-297. https://doi. org/10.3354/meps235289

Williams, S. L., Crafton, R. E., Fontana, R. E., Grosholz, E. D., Ha, G., Pasari, J. R. \& Zabin, C. J. (2015). A vector analysis of marine ornamental species in California. Manag. Biol. Invasions, 6(1), 13-29. https://doi.org/10.3391/ mbi.2015.6.1.02 
